OPEN ACCESS

Edited by:

Kriti Puri,

Baylor College of Medicine,

United States

Reviewed by:

Jun Lyu,

First Affiliated Hospital of Jinan

University, China

Dongfeng Zhang,

Qingdao University, China

${ }^{*}$ Correspondence:

Wenqi Song

songwenqi1218@163.com

Xiaoxia Peng

pengxiaoxia@bch.com.cn

${ }^{\dagger}$ These authors have contributed equally to this work

Specialty section

This article was submitted to Pediatric Cardiology,

a section of the journal

Frontiers in Pediatrics

Received: 20 May 2021

Accepted: 02 July 2021

Published: 09 August 2021

Citation:

Peng Y, Hu L, Nie X, Cai S, Yan R,

Liu $Y$, Cai $Y$, Song $W$ and Peng $X$

(2021) The Role of Serum Calcium Levels in Pediatric Dyslipidemia: Are

There Any? Front. Pediatr. 9:712160.

doi: 10.3389/fped.2021.712160

\section{The Role of Serum Calcium Levels in Pediatric Dyslipidemia: Are There Any?}

\author{
Yaguang Peng ${ }^{1}$, Lixin Hu${ }^{2}$, Xiaolu Nie ${ }^{1}$, Siyu Cai ${ }^{1}$, Ruohua Yan ${ }^{1}$, Yali Liu ${ }^{1}$, Yanying Cai ${ }^{2}$, \\ Wenqi Song ${ }^{2 *+}$ and Xiaoxia Peng ${ }^{1 *+}$ \\ ${ }^{1}$ Center for Clinical Epidemiology and Evidence-Based Medicine, National Center for Children's Health, Beijing Children's \\ Hospital, Capital Medical University, Beijing, China, ${ }^{2}$ Clinical Laboratory Center, National Center for Children's Health, Beijing \\ Children's Hospital, Capital Medical University, Beijing, China
}

Background: No previous study explored the association between serum calcium levels and dyslipidemia in children. This study aimed to explore this relationship in children, based on a multicenter cross-sectional study population in China.

Methods: Cross-sectional data was derived from the Pediatric Reference Intervals in China (PRINCE) study conducted between 2017 and 2018 involving 5,252 males and 5,427 females with a mean age of $10.0 \pm 4.6$ years. Multivariable logistic regression models were applied to calculate odds ratios (ORs), with 95\% confidence intervals (Cls), for dyslipidemia of each serum calcium level and albumin-corrected calcium levels, which were sorted into quartiles. The restricted cubic spline model was fitted for the dose-response analysis. An L-shaped dose-response relation between calcium levels and the probability of dyslipidemia was found after the adjustment for multiple potential confounding factors, $p$ for non-linear $<0.001$.

Results: Using the middle category of calcium level as the reference, multivariable-adjusted ORs and 95\% Cls of the lowest and the highest quartile categories were $0.96(0.82-1.12)$ and $1.29(1.12-1.48)$, respectively, for total serum calcium levels and $1.06(0.91-1.23)$ and 1.39 (1.21-1.60) for albumin-corrected calcium levels.

Conclusions: Individuals with higher levels of serum calcium were associated with increased risk of dyslipidemia in a sample of a healthy Chinese pediatric population. The association between serum calcium levels and dyslipidemia needs to be examined prospectively in future studies.

Keywords: calcium, dyslipidemia, pediatric, obesity, albumin-corrected calcium

\section{INTRODUCTION}

Calcium plays an important role in intracellular and extracellular signal transductions $(1,2)$ and muscle contractions (3), as well as other biological functions (4). Serum calcium level is mainly regulated by the balance of hormones (e.g., calcitonin and parathyroid hormone) and can be influenced by various factors such as diet, vitamin D levels, and daily physical activity (5-8). Recently, some epidemiological studies indicated that the serum calcium level was associated 
with cardiovascular diseases, including hypertension, diabetes, and insulin resistance in adult populations (9-12). Exposure to risk factors in childhood, such as abnormal glucose and lipid metabolism and obesity, increases the risk of cardiovascular metabolic disease in adulthood and has a persistent effect (1316). In the context of the COVID-19 pandemic, the risk factors of cardiovascular diseases in children and adolescents have attracted more attention due to changes in lifestyle factors including dietary nutrition, physical activity, sleep, sedentary, etc. (17, 18). The current situation of maternal and child healthcare in China were improved greatly, and the potential excessive use of calcium supplements and the coexistence of excessive nutrition and lack of exercise among children might increase the risk of cardiovascular diseases. Few studies have focused on the association between serum calcium levels and dyslipidemia in child populations, although the calcium homeostasis is vital for children's growth and development. In this study, we hypothesized that calcium levels might be associated with lipid metabolism in child populations. For this purpose, we analyzed the biochemical analyte data from the Pediatric Reference Intervals in China (PRINCE) study population.

\section{METHODS}

\section{Study Population}

Participants were derived from the PRINCE study enrolled from January 2017 to August 2018, which aimed to establish and verify pediatric reference intervals for Chinese children based on a nationwide multicenter cross-sectional study. A detailed description of the PRINCE study design and methods were published (19). Briefly, PRINCE was conducted by the Hospital Authority, National Health Commission of China. A major objective of PRINCE was to establish pediatric reference intervals (RIs) of 31 commonly used clinical laboratory parameters (18 measurands of complete blood count (CBC) and 13 biochemical analytes) based on a multicenter cross-sectional study covering 11 provinces and municipalities in China. Geographically, those centers covered the northeast (Liaoning Province), north (Beijing Municipality and Hebei Province), northwest (Shaanxi Province), middle (Henan and Hubei provinces), south (Guangdong Province), southwest (Chongqing Municipality and Sichuan Province), and east (Shanghai Municipality and Jiangsu Province) of China. The PRINCE study was composed of two phases: the establishment phase and the validation phase. The population of the establishment phase was involved in this study. In total, 15,150 participants aged 0-19 years old were screened in the PRINCE study. In this study, we only included individuals aged 2-18 years who completed the whole procedures including the epidemiology investigation, physical examination, blood sample collection, and laboratory test. After eliminating the missing values for critical variables, for instance serum calcium, serum lipid analytes, inorganic phosphate, albumin, resulted from the unqualified samples (e.g., insufficient sample volume, jaundice, hemolysis, or samples with chyle), 10,679 participants were included in the final analysis for this study. A detailed flow chart depicting participant selection is shown in Figure 1. The PRINCE study conformed to the ethical guidelines of the 1975 Declaration of Helsinki and was approved by The Institutional Review Board of Beijing Children's Hospital (IECC-028-A10-V.05). In addition, the protocol was approved by the institutional review boards of each collaborating centers. Informed consent was obtained from each participant's legal representative (parent or guardian) in the case of children aged 8 years or less. Additionally, it was assented by the child her/himself and consented by the legal guardian for children older than 8 years.

\section{Data Collection and Management}

In the PRINCE study, the Internet-based electronic data capture (EDC) platform was developed and used for data collection and management. Upon the completion of laboratory testing (CBC and biochemical markers), data managers at each center exported the results from the laboratory information system to the EDC platform. The whole data collection was completed after combining the epidemiology investigation, physical examination data, detailed information for sample collection and transport, and laboratory testing results. Some raw data was recorded in paper form when using the Internet was not convenient. All the paper forms were inputted and uploaded onto the platform, and the original data was stored and reviewed by the Data and Safety Monitoring Board (DSMB) at Beijing Children's Hospital. All data corrections and revisions will be tracked and marked.

Three sections of the data from the PRINCE database were extracted for this study: basic demographic information, epidemiology investigation data, and the laboratory test data of relevant analytes. Considering the age distribution and characteristics of analytes, five age subgroups were divided for this study, as such: $2-5,6-9,10-12,13-15$, and 16-18 years. The age- and sex-specific BMI Z-score were calculated according to the standard methods recommended by the World Health Organization $(20,21)$.

\section{Laboratory Tests and Clinical Definitions}

Participants in the PRINCE study were required to fast for at least $8 \mathrm{~h}$ before their blood sample collection, which occurred between 8 and 11 a.m., extracted by trained pediatric nurses using a BD Vacutainer and vacuum tube needles (Becton, Dickinson and Company, Dublin, Ireland). All blood sampling procedures to collect, handle, and store these samples were strictly in accordance with standard operating protocols (SOPs), which were reviewed and adopted by the committee of the PRINCE. After processing at each center, all the specimens were transported to the central laboratory at Beijing Children's Hospital in cold chain for analysis, which was completed within 6 months after collection, without repeated freeze-thaw cycles. The biomedical markers were measured using an automated biochemistry analyzer, Cobas C702 (Roche Diagnostics GmbH, Mannheim, Germany). The detailed procedure and quality control have been reported (19). The detection methods of biochemical analytes involved in this study were listed in Supplementary Table 1.

The diagnosis definition of dyslipidemia in children agreed upon by Chinese experts is as follows (22): (1) total cholesterol (TC) $\geq 5.18 \mathrm{mmol} / \mathrm{L}$, (2) triglyceride $(\mathrm{TG}) \geq 1.70 \mathrm{mmol} / \mathrm{L}$; (3) 


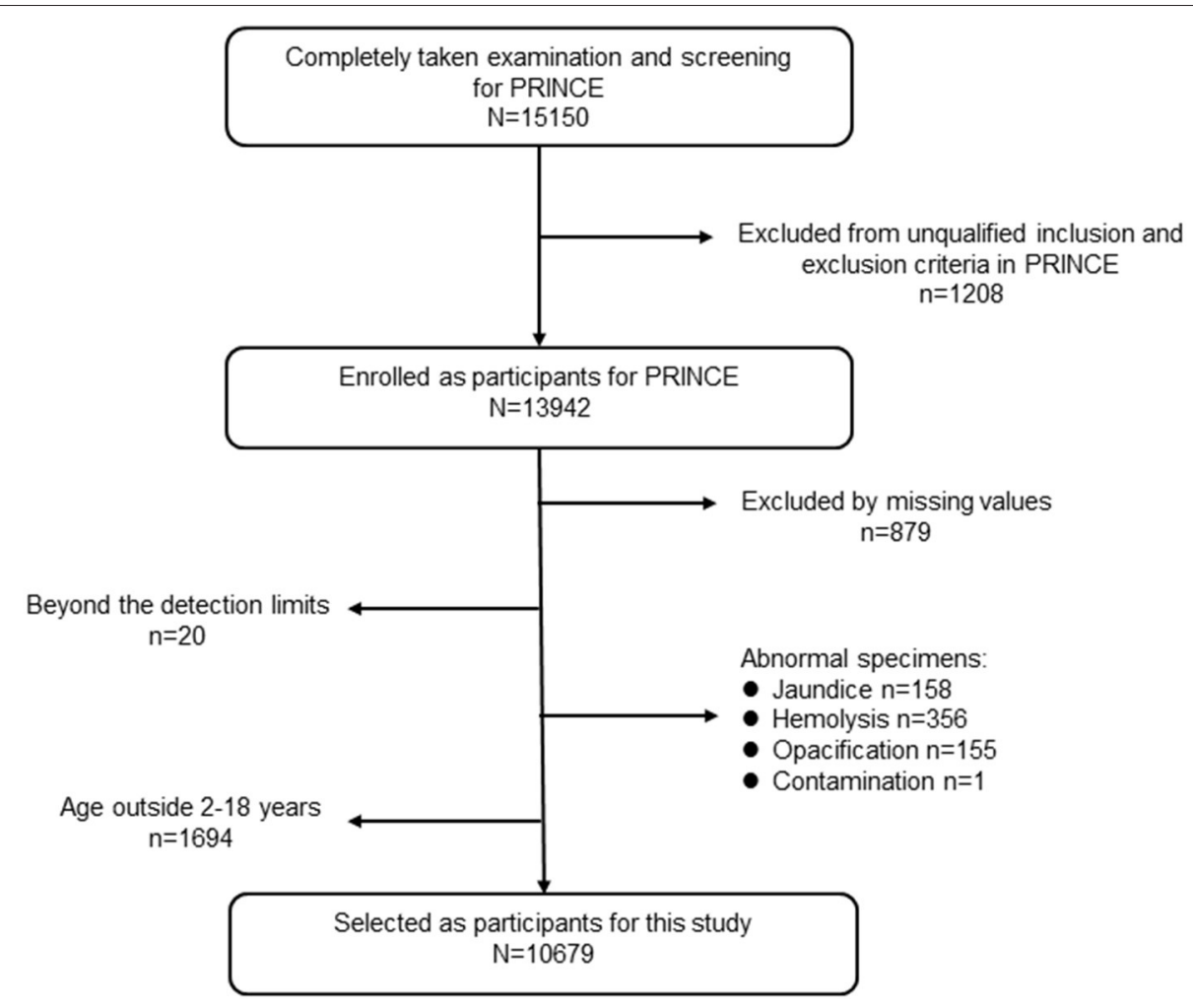

FIGURE 1 | The flowchart of the study population from the PRINCE study.

high-density lipoprotein-cholesterol (HDL-C) $\leq 1.04 \mathrm{mmol} / \mathrm{L}$; or (4) low-density lipoprotein-cholesterol (LDL-C) $\geq 3.37 \mathrm{mmol} / \mathrm{L}$. Glycemia was defined as fasting glucose $\geq 7.00 \mathrm{mmol} / \mathrm{L}$ according to the American Diabetes Association (23). Albumin-corrected calcium levels (24) were calculated as follows: serum calcium $(\mathrm{mmol} / \mathrm{L})+0.02 \times[40$-serum albumin $(\mathrm{g} / \mathrm{L})]$. Creatinine clearance $\left(\mathrm{ml} / \mathrm{min} / 1.73 \mathrm{~m}^{2}\right)$ was estimated by Schwartz formulas $(25,26)$.

\section{Statistical Analysis}

Data was depicted as a mean and standard deviation (SD) for continuous variables and as frequency (percentages) for categorical variables. Serum calcium levels and albumincorrected calcium levels were analyzed as categorical variables according to the 25th $\left(\mathrm{P}_{25}\right)$ and 75 th percentiles $\left(\mathrm{P}_{75}\right)$ of the distribution, respectively. Independent $t$-tests for continuous variables and $\chi^{2}$ tests for categorical variables were used to compare differences in characteristics between the dyslipidemia group and the control group. The middle group was defined as the reference and the lower $\mathrm{P}_{25}$ group and the higher $\mathrm{P}_{75}$ group were compared with middle group in order to explore the similar trends using the logistic analysis. The odds ratios (ORs) and 95\% confidence intervals (CIs) for dyslipidemia and its components of each calcium category were estimated using multivariate nonconditional logistic regression models. The status of dyslipidemia was taken as the dependent variable, and serum calcium and corrected calcium levels were taken as independent variables to fit multivariate models separately. Multivariate models 1, 2, and 3 were fitted successively considering different adjustment factors, including age, sex, BMI z-score, serum albumin levels, serum creatinine levels, alanine aminotransferase, inorganic phosphate, alkaline phosphatase, and total protein. The restricted cubic spline model was used for the dose-response analysis. Using serum-corrected calcium level as the dependent variable and age, height, weight BMI, serum albumin levels, serum creatinine levels, inorganic phosphate, alkaline phosphatase, total protein, etc. as the independent variables, the quality association between serum calcium level and related anthropometric indices and biomarkers were analyzed by multiple linear regression model. A two-tailed $p<0.05$ was considered significant. All statistics were conducted using SAS version 9.4 (SAS Institute, Cary, NC, USA) and $\mathrm{R}$ version 3.6.3 (R Foundation for Statistical Computing, Vienna, Austria).

\section{RESULTS}

The characteristics of this population are depicted in Table 1. There were a total of 10,679 participants, 5,252 males and 5,427 females, with a mean age of $10.0 \pm 4.6$ years. The dyslipidemia rate was $12.7 \%$ in males and $13.6 \%$ in females, respectively. The sex-specific rates of components, high TC, high TG, high LDLC, and low HDL-C were 3.5, 3.2, 4.1, and 5.1\% in males and 4.3, $4.2,4.8$, and $4.3 \%$ in females. With exception of the high LDL-C rates with $p$-value 0.053 , other differences above between sexes 
TABLE 1 | Characteristics of the study population $(n=10,679)$.

\begin{tabular}{|c|c|c|c|c|c|c|c|c|c|c|}
\hline & \multicolumn{5}{|c|}{ Female } & \multicolumn{5}{|c|}{ Male } \\
\hline & $2-5$ years & $6-9$ years & $10-12$ years & $13-15$ years & $16-18$ years & $2-5$ years & $6-9$ years & $10-12$ years & $13-15$ years & $16-18$ years \\
\hline $\mathrm{N}$ & 1,164 & 1,458 & 1,041 & 910 & 854 & 1,311 & 1,547 & 1,081 & 788 & 525 \\
\hline Age, year & $4.00 \pm 1.11$ & $8.04 \pm 1.13$ & $11.47 \pm 0.90$ & $14.53 \pm 0.92$ & $17.48 \pm 0.87$ & $3.91 \pm 1.10$ & $8.02 \pm 1.12$ & $11.48 \pm 0.90$ & $14.48 \pm 0.92$ & $17.30 \pm 0.85$ \\
\hline Height, cm & $102.67 \pm 9.52$ & $128.52 \pm 8.90$ & $149.28 \pm 9.14$ & $159.23 \pm 6.38$ & $160.77 \pm 6.08$ & $103.30 \pm 9.40$ & $129.41 \pm 9.08$ & $149.14 \pm 10.07$ & $167.14 \pm 8.72$ & $172.91 \pm 6.54$ \\
\hline Weight, kg & $16.53 \pm 3.59$ & $27.01 \pm 6.43$ & $41.13 \pm 10.06$ & $51.98 \pm 9.70$ & $55.48 \pm 8.57$ & $17.00 \pm 3.61$ & $28.83 \pm 7.96$ & $43.80 \pm 12.77$ & $57.80 \pm 13.51$ & $64.81 \pm 12.34$ \\
\hline $\mathrm{BMl}, \mathrm{kg} / \mathrm{m}^{2}$ & $15.55 \pm 1.66$ & $16.18 \pm 2.44$ & $18.28 \pm 3.06$ & $20.45 \pm 3.33$ & $21.45 \pm 2.89$ & $15.81 \pm 1.64$ & $17.00 \pm 3.26$ & $19.41 \pm 4.05$ & $20.53 \pm 3.84$ & $21.67 \pm 3.68$ \\
\hline BMl z-score & $0.06 \pm 1.10$ & $0.02 \pm 1.16$ & $0.06 \pm 1.14$ & $0.03 \pm 1.04$ & $0.00 \pm 0.87$ & $0.19 \pm 1.19$ & $0.41 \pm 1.52$ & $0.57 \pm 1.43$ & $0.18 \pm 1.24$ & $-0.07 \pm 1.22$ \\
\hline Total protein, $\mathrm{g} / \mathrm{L}$ & $69.82 \pm 3.77$ & $73.61 \pm 3.83$ & $75.17 \pm 3.94$ & $78.08 \pm 4.25$ & $77.52 \pm 3.99$ & $69.50 \pm 3.61$ & $73.25 \pm 3.74$ & $75.22 \pm 3.73$ & $77.17 \pm 4.06$ & $77.02 \pm 3.87$ \\
\hline Albumin, $g / L$ & $47.64 \pm 2.27$ & $48.87 \pm 2.38$ & $49.34 \pm 2.39$ & $50.49 \pm 2.53$ & $50.17 \pm 2.35$ & $47.47 \pm 2.23$ & $48.45 \pm 2.26$ & $49.53 \pm 2.38$ & $50.97 \pm 2.66$ & $51.34 \pm 2.37$ \\
\hline Creatinine, $\mu \mathrm{mol} / \mathrm{L}$ & $32.80 \pm 5.38$ & $42.36 \pm 5.77$ & $47.58 \pm 7.45$ & $57.13 \pm 8.81$ & $61.08 \pm 8.13$ & $33.20 \pm 5.45$ & $43.40 \pm 6.20$ & $51.43 \pm 7.61$ & $67.32 \pm 12.65$ & $79.64 \pm 11.06$ \\
\hline Alanine aminotransferase, U/L & $13.46 \pm 4.63$ & $13.16 \pm 5.99$ & $12.94 \pm 6.66$ & $12.38 \pm 5.85$ & $12.76 \pm 7.31$ & $14.05 \pm 7.78$ & $14.78 \pm 13.02$ & $16.34 \pm 14.59$ & $16.36 \pm 14.69$ & $18.68 \pm 15.21$ \\
\hline Aspartate aminotransferase, U/L & $29.56 \pm 5.59$ & $24.65 \pm 4.54$ & $21.10 \pm 6.81$ & $18.12 \pm 3.90$ & $18.33 \pm 10.32$ & $30.14 \pm 6.47$ & $25.52 \pm 7.43$ & $23.13 \pm 7.14$ & $21.50 \pm 21.56$ & $22.19 \pm 33.48$ \\
\hline Serum calcium, $\mathrm{mmol} / \mathrm{L}$ & $2.52 \pm 0.08$ & $2.50 \pm 0.07$ & $2.48 \pm 0.07$ & $2.48 \pm 0.08$ & $2.46 \pm 0.08$ & $2.51 \pm 0.09$ & $2.48 \pm 0.07$ & $2.49 \pm 0.07$ & $2.49 \pm 0.08$ & $2.49 \pm 0.08$ \\
\hline Albumin-corrected calcium, $\mathrm{mmol} / \mathrm{L}$ & $2.36 \pm 0.07$ & $2.32 \pm 0.06$ & $2.29 \pm 0.07$ & $2.27 \pm 0.07$ & $2.26 \pm 0.07$ & $2.36 \pm 0.07$ & $2.31 \pm 0.06$ & $2.30 \pm 0.06$ & $2.27 \pm 0.07$ & $2.26 \pm 0.07$ \\
\hline Inorganic phosphate, $\mathrm{mmol} / \mathrm{L}$ & $1.68 \pm 0.13$ & $1.60 \pm 0.14$ & $1.57 \pm 0.18$ & $1.36 \pm 0.17$ & $1.29 \pm 0.14$ & $1.68 \pm 0.14$ & $1.60 \pm 0.15$ & $1.60 \pm 0.16$ & $1.49 \pm 0.24$ & $1.26 \pm 0.19$ \\
\hline Alkaline phosphatase, $\mathrm{mmol} / \mathrm{L}$ & $230.40 \pm 61.54$ & $245.27 \pm 62.34$ & $262.76 \pm 79.51$ & $137.97 \pm 64.02$ & $78.21 \pm 24.44$ & $225.24 \pm 61.24$ & $238.37 \pm 56.21$ & $287.74 \pm 85.97$ & $252.91 \pm 110.43$ & $117.21 \pm 48.65$ \\
\hline Creatinine clearance, $\mathrm{ml} / \mathrm{min} / 1.73 \mathrm{~m}^{2}$ & $155.07 \pm 21.78$ & $149.78 \pm 19.57$ & $155.50 \pm 22.61$ & $138.44 \pm 21.42$ & $130.03 \pm 17.05$ & $154.21 \pm 21.61$ & $147.65 \pm 22.47$ & $143.37 \pm 18.87$ & $158.40 \pm 26.86$ & $136.70 \pm 19.3$ \\
\hline Triglyceride, $\mathrm{mmol} / \mathrm{L}$ & $0.80 \pm 0.31$ & $0.81 \pm 0.32$ & $1.00 \pm 0.42$ & $1.02 \pm 0.43$ & $0.95 \pm 0.40$ & $0.74 \pm 0.30$ & $0.77 \pm 0.34$ & $0.90 \pm 0.42$ & $0.95 \pm 0.43$ & $0.91 \pm 0.39$ \\
\hline Total cholesterol, mmol/L & $4.03 \pm 0.72$ & $3.98 \pm 0.68$ & $3.88 \pm 0.64$ & $3.86 \pm 0.68$ & $3.74 \pm 0.66$ & $3.95 \pm 0.70$ & $3.97 \pm 0.69$ & $3.89 \pm 0.65$ & $3.58 \pm 0.63$ & $3.53 \pm 0.60$ \\
\hline Low density lipoprotein cholesterin, $\mathrm{mmol} / \mathrm{L}$ & $2.48 \pm 0.65$ & $2.31 \pm 0.61$ & $2.20 \pm 0.58$ & $2.17 \pm 0.60$ & $2.11 \pm 0.57$ & $2.37 \pm 0.63$ & $2.27 \pm 0.62$ & $2.24 \pm 0.62$ & $1.97 \pm 0.57$ & $2.04 \pm 0.52$ \\
\hline High density lipoprotein cholesterin, mmol/L & $1.48 \pm 0.32$ & $1.58 \pm 0.33$ & $1.52 \pm 0.31$ & $1.49 \pm 0.30$ & $1.51 \pm 0.31$ & $1.53 \pm 0.33$ & $1.64 \pm 0.35$ & $1.55 \pm 0.34$ & $1.40 \pm 0.31$ & $1.34 \pm 0.29$ \\
\hline Blood glucose, $\mathrm{mmol} / \mathrm{L}$ & $4.49 \pm 0.50$ & $4.63 \pm 0.50$ & $4.69 \pm 0.46$ & $4.75 \pm 0.48$ & $4.75 \pm 0.72$ & $4.61 \pm 0.49$ & $4.75 \pm 0.52$ & $4.76 \pm 0.47$ & $4.82 \pm 0.52$ & $4.65 \pm 0.55$ \\
\hline Dyslipidemia & $207(17.78)$ & $142(9.74)$ & $148(14.22)$ & $145(15.93)$ & $97(11.36)$ & $167(12.74)$ & $159(10.28)$ & $136(12.58)$ & $121(15.36)$ & $85(16.19)$ \\
\hline High TG, $n(\%)$ & $21(1.80)$ & $25(1.71)$ & $69(6.63)$ & $73(8.02)$ & $38(4.45)$ & $17(1.30)$ & $26(1.68)$ & $56(5.18)$ & $47(5.96)$ & $22(4.19)$ \\
\hline High TC, $n(\%)$ & $70(6.01)$ & $64(4.39)$ & 39 (3.75) & $35(3.85)$ & $27(3.16)$ & $54(4.12)$ & $81(5.24)$ & $34(3.15)$ & $10(1.27)$ & $4(0.76)$ \\
\hline High LDL-C, $n(\%)$ & $101(8.68)$ & $68(4.66)$ & $31(2.98)$ & 32 (3.52) & $30(3.51)$ & $77(5.87)$ & 75 (4.85) & $45(4.16)$ & $7(0.89)$ & $9(1.71)$ \\
\hline Low HDL-C, $n(\%)$ & $73(6.27)$ & $44(3.02)$ & $49(4.71)$ & $37(4.07)$ & $29(3.40)$ & $62(4.73)$ & $38(2.46)$ & $40(3.70)$ & $76(9.64)$ & $52(9.90)$ \\
\hline
\end{tabular}

TG, triglyceride; TC, total cholesterol; LDL-C, low density lipoprotein-cholesterol; HDL-C, high density lipoprotein-cholesterol. 
TABLE 2 | Comparisons between normality and dyslipidemia.

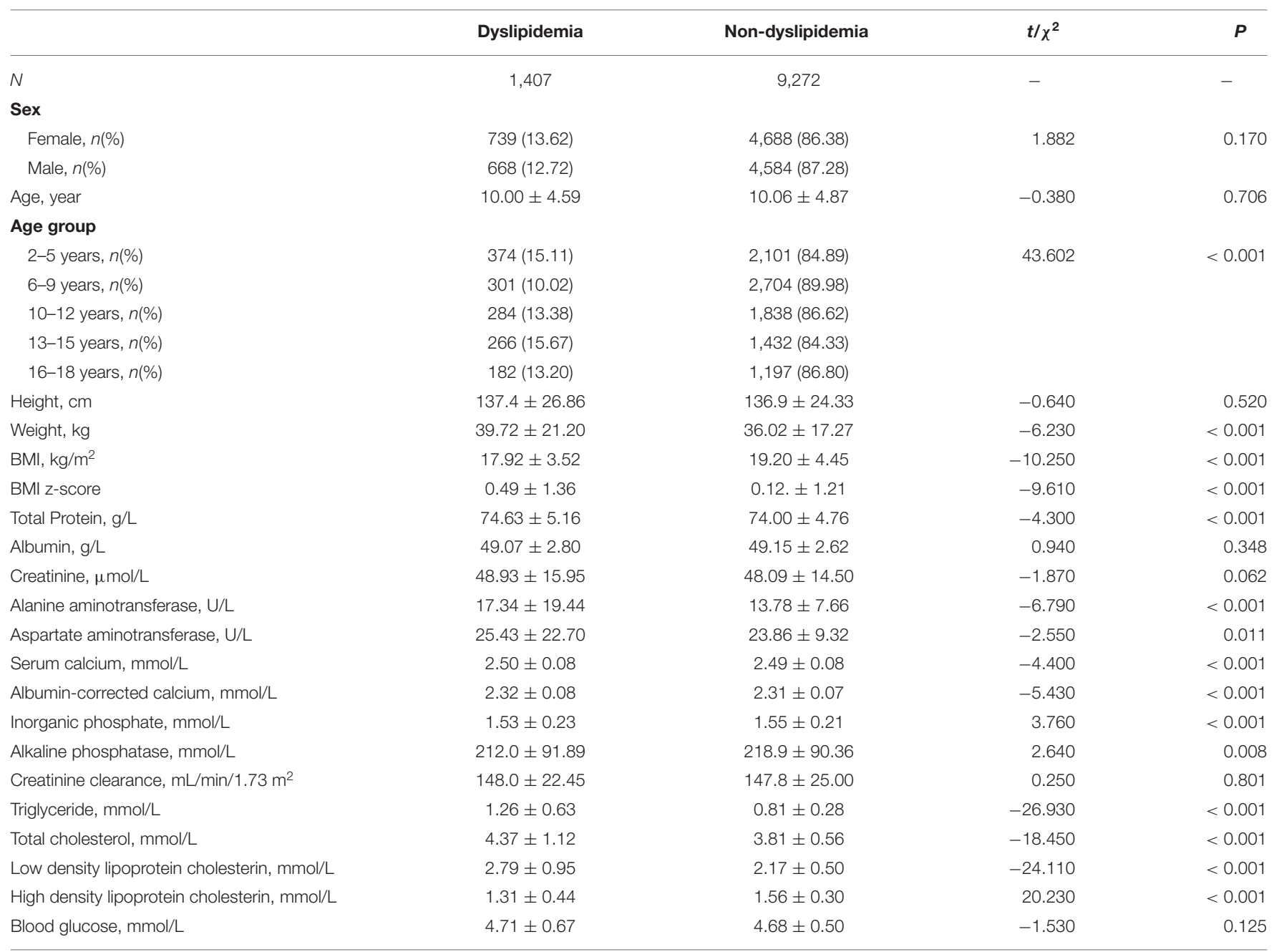

were significant ( $p$-values were $0.024,0.008$, and 0.043 for high TC, high TG, and low HDL-C). Biochemical analytes in this study indicated the age-dependent trend.

Table 2 presents the differences between healthy children and those with dyslipidemia. The differences in albumin, creatinine, creatinine clearance, and blood glucose were non-significant. The serum calcium and albumin-corrected calcium level in those with dyslipidemia were higher than those in healthy children.

Based on the distribution of the serum calcium and albumincorrected calcium, three categories were divided. The 25 th and 75 th percentile were 2.44 and $2.54 \mathrm{mmol} / \mathrm{L}$ for serum calcium levels and 2.25 and $2.54 \mathrm{mmol} / \mathrm{L}$ for albumin-corrected calcium levels. An increasing tendency in the rate of dyslipidemia both in serum calcium level and albumin-corrected calcium was observed, which was only statistically significant for albumincorrected calcium ( $p$ for trend $<0.001$ ). Except for high TG, a significant monotonic trend was revealed between dyslipidemia components and calcium levels (all $p$ for trend $<0.001$ ). The rate of high TC and high LDL-C increased with calcium levels, while low HDL-C decreases (Table 3). Similarly, the monotonic trends were found in the multivariable analysis.
In the age-, sex-, and BMI z-score-adjusted model (Model 1 ), the highest categories of serum calcium and albumincorrected calcium levels were both associated with higher probabilities for dyslipidemia, using the middle category as the reference. This association was not altered after further adjustment for several other covariates and confounding factors including albumin, alanine aminotransferase, creatinine, inorganic phosphate, alkaline phosphatase, and total protein. With the middle category of serum calcium level as the reference, the multivariable-adjusted ORs and 95\% CIs of the lowest and the highest categories were $0.96(0.82-1.12)$ and $1.29(1.12-$ $1.48)$, respectively, for total serum calcium levels and 1.06 (0.91$1.23)$ and 1.39 (1.21-1.60) for albumin-corrected calcium levels (Table 4). The standardized coefficients of TC, TG, LDL-C, and HDL-C for albumin-corrected calcium levels were 0.411, 0.031, -0.270 , and -0.045 (all $p$-values $<0.05$ ) in the linear regression model (Supplementary Table 2).

The restricted cubic spline model indicated an L-shaped dose-response relation between serum calcium levels and the probability of dyslipidemia after adjustment for multiple potential confounding factors ( $p$ for nonlinear <0.001) 
TABLE 3 | The rate of dyslipidemia and components trends with serum calcium and albumin corrected calcium.

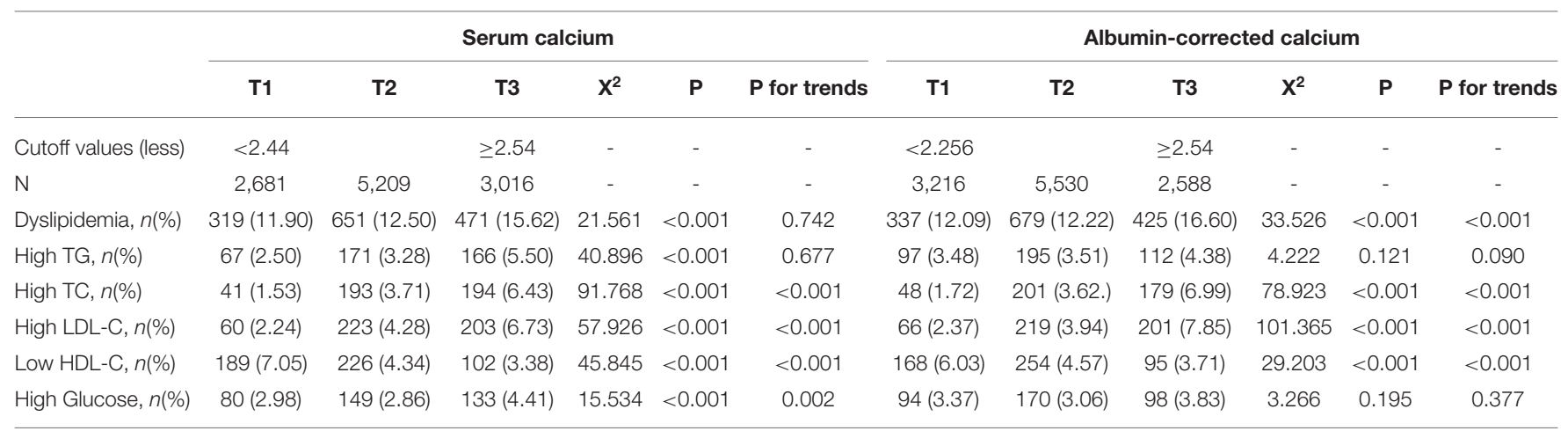

Calcium categories were $<2.44,2.44-2.53$, and $\geq 2.54 \mathrm{mmol} / \mathrm{L}$; Albumin corrected calcium categories were $<2.26,2.26-2.53$, and $\geq 2.54 \mathrm{mmol} / \mathrm{L}$.

TG, triglyceride; TC, total cholesterol; LDL-C, low density lipoprotein-cholesterol; HDL-C, high density lipoprotein-cholesterol.

TABLE 4 | Multivariable-adjusted odds ratios and 95\% confidence intervals of serum calcium and albumin corrected calcium associated with dyslipidemia.

\begin{tabular}{|c|c|c|c|c|c|c|c|}
\hline & & \multicolumn{3}{|c|}{ Serum calcium } & \multicolumn{3}{|c|}{ Albumin-corrected calcium } \\
\hline & & T1 & T2 & T3 & T1 & T2 & T3 \\
\hline & High TG & $0.755(0.567-1.006)$ & Ref & 1.716 (1.379-2.135) & $0.991(0.774-1.270)$ & Ref & $1.258(0.993-1.595)$ \\
\hline & High TC & $0.404(0.287-0.567)$ & Ref & 1.787 (1.456-2.192) & $0.467(0.340-0.642)$ & Ref & $2.004(1.629-2.465)$ \\
\hline & High LDL-C & $0.512(0.383-0.683)$ & Ref & 1.614 (1.327-1.962) & $0.591(0.447-0.781)$ & Ref & $2.077(1.705-2.531)$ \\
\hline & High Gluc & $1.045(0.793-1.376)$ & Ref & $1.567(1.235-1.988)$ & $1.106(0.856-1.429)$ & Ref & $1.262(0.980-1.625)$ \\
\hline \multirow[t]{4}{*}{ Adjusted Model 1} & Dyslipidemia & $0.978(0.844-1.134)$ & Ref & $1.271(1.115-1.449)$ & $0.945(0.814-1.096)$ & Ref & $1.487(1.293-1.711)$ \\
\hline & High TG & $0.706(0.524-0.950)$ & Ref & $1.800(1.436-2.256)$ & $0.734(0.565-0.954)$ & Ref & $1.828(1.416-2.361)$ \\
\hline & High TC & $0.421(0.296-0.600)$ & Ref & $1.702(1.379-2.100)$ & $0.467(0.331-0.659)$ & Ref & $1.865(1.492-2.331)$ \\
\hline & High LDL-C & $0.561(0.416-0.757)$ & Ref & $1.474(1.205-1.803)$ & $0.653(0.482-0.885)$ & Ref & $1.732(1.402-2.139)$ \\
\hline \multirow{4}{*}{ Adjusted Model 2} & High TC & $0.440(0.307-0.632)$ & Ref & $1.629(1.300-2.042)$ & $0.460(0.326-0.650)$ & Ref & $1.875(1.498-2.347)$ \\
\hline & High LDL-C & $0.556(0.408-0.757)$ & Ref & $1.490(1.201-1.848)$ & $0.657(0.485-0.891)$ & Ref & $1.723(1.394-2.129)$ \\
\hline & Low HDL-C & $1.520(1.220-1.893)$ & Ref & $0.863(0.668-1.115)$ & $1.532(1.231-1.907)$ & Ref & $0.763(0.592-0.983)$ \\
\hline & High Gluc & $0.891(0.662-1.200)$ & Ref & $1.711(1.322-2.215)$ & $0.830(0.632-1.089)$ & Ref & $1.759(1.344-2.303)$ \\
\hline \multirow[t]{6}{*}{ Adjusted Model 3} & Dyslipidemia & $0.956(0.817-1.118)$ & Ref & $1.288(1.117-1.484)$ & $1.056(0.907-1.231)$ & Ref & $1.390(1.206-1.603)$ \\
\hline & High TG & $0.818(0.598-1.119)$ & Ref & $1.554(1.212-1.994)$ & $0.832(0.636-1.088)$ & Ref & $1.685(1.297-2.189)$ \\
\hline & High TC & $0.464(0.322-0.667)$ & Ref & $1.513(1.203-1.901)$ & $0.494(0.349-0.700)$ & Ref & $1.765(1.407-2.214)$ \\
\hline & High LDL-C & $0.573(0.420-0.781)$ & Ref & $1.398(1.124-1.739)$ & $0.688(0.505-0.936)$ & Ref & $1.637(1.322-2.028)$ \\
\hline & Low HDL-C & $1.547(1.237-1.933)$ & Ref & $0.822(0.635-1.065)$ & $1.585(1.269-1.980)$ & Ref & $0.739(0.573-0.954)$ \\
\hline & High Gluc & $0.875(0.648-1.182)$ & Ref & $1.696(1.307-2.203)$ & $0.824(0.625-1.086)$ & Ref & $1.763(1.343-2.314)$ \\
\hline
\end{tabular}

Model 1 adjusted for age, sex, and BMI z-score (continuous).

Model 2 further adjusted for albumin (continuous), alanine aminotransferase (continuous), and creatinine (continuous).

Model 3 further adjusted for inorganic phosphate (continuous), alkaline phosphatase (continuous), and total protein (continuous).

Calcium categories were $<2.44,2.44-2.53$, and $\geq 2.54 \mathrm{mmol} / \mathrm{L}$; Albumin corrected calcium categories were $<2.26,2.26-2.53$, and $\geq 2.54 \mathrm{mmo} / \mathrm{L}$.

TG, triglyceride; TC, total cholesterol; LDL-C, low density lipoprotein-cholesterol; HDL-C, high density lipoprotein-cholesterol; Ref, reference.

(Figure 2A). However, the risk of dyslipidemia only showed a monotonically increasing tendency when the albumin-corrected calcium level was beyond $2.50 \mathrm{mmol} / \mathrm{L}$ after adjustment of confounding factors as well $(p$ for nonlinear <0.001) (Figure 2B).

\section{DISCUSSION}

The physiological function of calcium is to not only participate in osteogenesis and function within osteoclasts but is also involved in neurotransmitter release, hormone secretion, maintenance of 


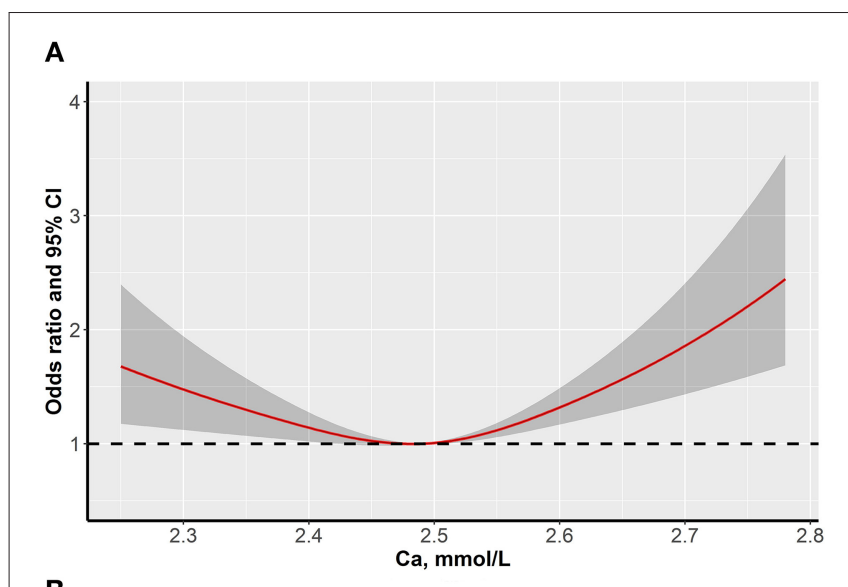

B

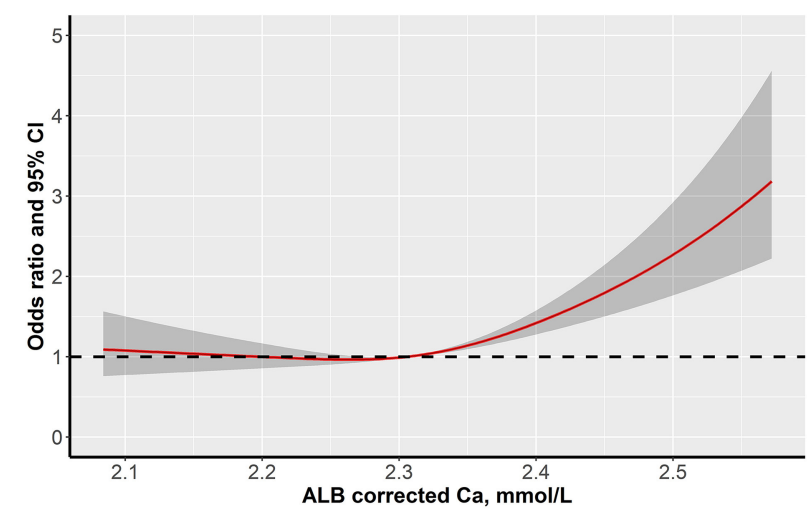

FIGURE 2 | Dose-response associations between serum calcium levels and dyslipidemia (A is serum calcium levels and $\mathbf{B}$ is albumin-corrected calcium levels).

blood coagulation, and homeostasis (12). In plasma, about $50 \%$ of calcium is free or ionized, $40 \%$ is bound to plasma proteins, and $10 \%$ is complexed with anions such as bicarbonate, phosphate, lactate, and citrate (4). Albumin is the predominant calciumbinding protein with $1 \mathrm{~g} / \mathrm{L}$ of albumin binding to approximately $0.8 \mathrm{mg} / \mathrm{dl}$ of calcium (24).

Epidemiological studies found that higher serum calcium levels and albumin-corrected calcium levels were associated with an increased risk of developing metabolic syndrome, diabetes, and hypertension $(6,27-29)$.

The US National Cholesterol Education Program (NCEP) proposed a set of cut-off values for lipid concentrations to define dyslipidemia aged 2-19 years. The definition of dyslipidemia based on Chinese expert consensus in this study has a little bit difference from the NCEP pediatric cutpoints, which was the same as in high TC $(\geq 5.18 \mathrm{mmol} / \mathrm{L})$ and high LDL-C $(\geq 3.37$ $\mathrm{mmol} / \mathrm{L})$, but higher in low HDL-C $(\leq 1.04 \mathrm{mmol} / \mathrm{L}$ in China vs. $\leq 0.91 \mathrm{mmol} / \mathrm{L}$ in US $)$ and high TG $(\geq 1.70 \mathrm{mmol} / \mathrm{L}$ in China vs. $\geq 1.13 \mathrm{mmol} / \mathrm{L}$ for $2-9$ years and $\geq 1.47 \mathrm{mmol} / \mathrm{L}$ for $10-19$ years in the USA) $(22,23)$.

The results from Tables 3,4 show that among several indicators of dyslipidemia, there was no statistical significance in high TG rates among percentiles of calcium levels, whether using the total serum calcium level or albumin-corrected levels.

A similar trend was also confirmed in multivariable models. All other biomarkers of dyslipidemia separately depicted linear increasing trends. This difference in cholesterol and triglyceride associated with calcium could be caused by the distinctions of metabolism and regulation of triglyceride and cholesterol. The former is regulated by hormones, such as insulin, while the latter is regulated by lipoproteins.

In a recent epidemiological investigation of osteoporosis prevention in postmenopausal women, long-term calcium supplementation could affect the serum cholesterol level of a female population. Another research revealed high calcium intake increases in rat serum levels of TC, TG, LDL-C, and HDL$\mathrm{C}$ and eventually leads to calcium depositions in the carotid artery (30). The association between calcium intakes and lipid metabolism had been found in both animal researches and population studies. The mechanism was thought to be that longterm calcium supplementation converted the smooth muscle cells into osteoblast-like cells, which caused the formation of matrix vesicles, which secrete mineralized crystals, leading to smooth muscle cell apoptosis (31).

Studies showed that fluctuating calcium concentrations could reflect not only the exogenous intake but also the endogenous capability of maintaining homeostasis (32), which was tightly regulated by multiple negative feedback loops involving several target organs and hormones (33). Another study observed a significant correlation between serum calcium levels and TC, HDL-C, LDL-C, non-HDL-C, and TG both in postmenopausal women and in men (34). The potential mechanisms involved in this were hypothesized but remain unclear. Firstly, higher serum calcium levels resulted in smaller concentrations of serum pyrophosphates and greater tissue calcification (6). A study explored the effect of blood lipid markers on nephrolithiasis in children; it suggested that children with calcium oxalate stones accompanied with the existence of abnormal lipid metabolism (35).

Secondly, mitochondrial and free calcium dynamics formed a homeostatic circuit that compartmentalized and coordinated energy metabolism, ROS production, signal transduction, proliferation, and cell death due to internal and environmental cues $(2,36,37)$. It was confirmed that serum calcium levels were directly correlated to glucose intolerance, insulin resistance, impaired glucose metabolism, early-phase insulin secretion, and diabetes (12). All of the aforementioned factors went hand in hand with dyslipidemia. Another explanation indicated the influence of hepatic catabolism, especially in estrogen-deficient conditions (38). Presence of calcium could decrease cholesterol catabolism, and stimulates lipids synthesis. In more details, higher calcium levels could decrease the activity of the 7a-hydroxylase (CYP7A), an enzyme involved in the cholesterol catabolism, and stimulates sterol regulatory element-binding protein (SREBP)-1c expression, which is a transcription factor involved in de novo lipid synthesis (39).

In addition, the regulation of calcitonin, vitamin $\mathrm{D}$, and the parathyroid hormone might affect lipid metabolism as well. All 
hypotheses mentioned above would be explored and tested in further experiments.

Our study results suggested that serum calcium or albumincorrected calcium levels above $2.50 \mathrm{mmol} / \mathrm{L}$ in children might seem to be associated with the risk of dyslipidemia, although they failed to meet the clinical diagnosis cutoff value for hypercalcemia. Under the condition of the uncertain association, arbitrarily overuse of calcium, VD supplements, and the coexistence of excessive nutrition and lack of exercise among children on top of the epidemic period, all these would increase the risk of cardiovascular disease in children. Further clinical and basic researches are needed to clarify the mechanisms involved. If such association does exist and the mechanism should be clarified, it might lead to new targets for the clinical treatment of dyslipidemia, as well as to more rational and scientific considerations for the use of calcium supplements in children from a public health perspective.

Our study depicted an interesting phenomenon that there might be a potential association between serum calcium levels and dyslipidemia in a large and nationally representative sample of Chinese children. Nonetheless, this study had several limitations. Firstly, the cross-sectional nature of the PRINCE study made it difficult to determine a causal relationship between serum calcium level and dyslipidemia. The primary objective of the PRINCE study was to establish the pediatric reference intervals involved. Thus, the association between serum calcium level and dyslipidemia was still not clear whether it would exist in the natural population from community or in the case-control study, which would be also the direction for further studies to confirm it.

Secondly, due to the lack of physical activity data, dietary investigation including calcium intakes or supplement, and other relevant hormones or biomarkers (e.g., vitamin D, calcitonin, and parathyroid hormone), we cannot further analyze their effects on both, serum calcium levels and lipid metabolism. Further epidemiological or clinical studies that would take into account the corresponding factors should be conducted for validation purposes in the future.

Lastly, the continuous age-dependent trends in children should have been considered; the standardized deviation score (SDS) methods, which would adjust the influence by age and sex, could be applied when exploring the association between calcium levels and dyslipidemia. Further research in community

\section{REFERENCES}

1. Reid IR, Birstow SM, Bolland MJ. Calcium and cardiovascular disease. Endocrinol Metab. (2017) 32:339-49. doi: 10.3803/EnM.2017.32.3.339

2. Bravo-Sagua R, Parra V, López-Crisosto C, Díaz P, Quest AF, Lavandero S. Calcium transport and signaling in mitochondria. Compr Physiol. (2017) 7:623-34. doi: 10.1002/cphy.c160013

3. Park B, Kim MH, Cha CK, Lee YJ, Kim KC. High calcium-magnesium ratio in hair is associated with coronary artery calcification in middleaged and elderly individuals. Biol Trace Elem Res. (2017) 179:52-8. doi: 10.1007/s12011-017-0956-8

4. Song L. Calcium and bone metabolism indices. Adv Clin Chem. (2017) 82:1-46. doi: 10.1016/bs.acc.2017.06.005 child populations that focus on calcium SDS and other potential confounding factors should be designed and carried out.

\section{CONCLUSION}

We found that individuals with higher levels of serum calcium were associated with increased risk of dyslipidemia in a healthy sample of Chinese children. It is well-established that calcium deficiency is linked to some diseases and should be treated with calcium fortification or supplementation such as vitamin D supplements. However, potential adverse health outcomes, such as change in lipid metabolism and vascular calcification can be associated with high calcium volumes resulting from excessive supplementation and need to be explored in future studies.

\section{DATA AVAILABILITY STATEMENT}

The raw data supporting the conclusions of this article will be made available by the authors, without undue reservation.

\section{AUTHOR CONTRIBUTIONS}

$\mathrm{XP}$ and WS conceived the present study idea. YP, LH, XN, SC, RY, $\mathrm{YL}$, and YC conducted the study. YP, XN, SC, and RY conducted the data collection and data manage. LH and YC performed quality control of the sample collection and laboratory tests. YP and RY performed the data analysis. YP drafted the manuscript. All authors reviewed and approved the final manuscript and took responsibility for the reliability and freedom from bias of the data presented and their interpretation.

\section{FUNDING}

This study was supported by a project authorized by the National Health Commission of China (2017-374) and Beijing Municipal Administration of Hospital Clinical Medicine Development Special Project (ZYLX201840). The funding sources above had no role in the design, analysis, or writing of this manuscript.

\section{SUPPLEMENTARY MATERIAL}

The Supplementary Material for this article can be found online at: https://www.frontiersin.org/articles/10.3389/fped. 2021.712160/full\#supplementary-material

5. Norman PE, Powell JT. Vitamin D and cardiovascular disease. Circ Res. (2014) 114:379-93. doi: 10.1161/CIRCRESAHA.113.301241

6. Park B, Lee YJ. Borderline high serum calcium levels are associated with arterial stiffness and 10-year cardiovascular disease risk determined by Framingham risk score. J Clin Hypertens. (2019) 21:668-73. doi: $10.1111 /$ jch.13532

7. Underland L, Markowitz M, Gensure R. Calcium and phosphate hormones: vitamin d, parathyroid hormone, and fibroblast growth factor 23. Pediatr Rev. (2020) 41:3-11. doi: 10.1542/pir.2018-0065

8. Weaver CM, Peacock M. Calcium. Adv Nutr. (2019) 10:546-8. doi: 10.1093/advances/nmy086

9. Lv Y, Xie L, Dong C, Yang R, Long T, Yang H, et al. Co-exposure of serum calcium, selenium and vanadium is nonlinearly associated with increased risk 
of type 2 diabetes mellitus in a Chinese population. Chemosphere. (2021) 263:128021. doi: 10.1016/j.chemosphere.2020.128021

10. Wu X, Han T, Gao J, Zhang Y, Zhao S, Sun R, et al. Association of serum calcium and insulin resistance with hypertension risk: a prospective population-based study. J Am Heart Assoc. (2019) 8:e009585. doi: 10.1161/JAHA.118.009585

11. Kunutsor SK, Laukkanen JA. Circulating active serum calcium reduces the risk of hypertension. Eur J Prev Cardiol. (2017) 24:239-43. doi: $10.1177 / 2047487316681174$

12. Zhu J, Xun P, Bae JC, Kim JH, Kim DJ, Yang K, et al. Circulating calcium levels and the risk of type 2 diabetes: a systematic review and meta-analysis. $\mathrm{Br} \mathrm{J}$ Nutr. (2019) 122:376-87. doi: 10.1017/S0007114519001430

13. Umer A, Kelley GA, Cottrell LE, Giacobbi P Jr, Innes KE, Lilly CL. Childhood obesity and adult cardiovascular disease risk factors: a systematic review with meta-analysis. BMC Public Health. (2017) 17:683. doi: 10.1186/s12889-017-4691-z

14. Leyvraz M, Chatelan A, da Costa BR, Taffe P, Paradis G, Bovet P, et al. Sodium intake and blood pressure in children and adolescents: a systematic review and meta-analysis of experimental and observational studies. Int J Epidemiol. (2018) 47:1796-810. doi: 10.1093/ije/dyy121

15. Chen FF, Chang SY, Hou DQ, Gao AY, Zhu ZX, Yu ZC, et al. Characteristics of cardiovascular health of children and adolescents aged 6-16 years in Beijing during 2017-2018. Zhonghua Yu Fang Yi Xue Za Zhi. (2018) 52:1124-9. doi: 10.3760/cma.j.issn.0253-9624.2018.11.006

16. Lobstein T, Jackson-Leach R, Moodie ML, Hall KD, Gortmaker SL, Swinburn BA, et al. Child and adolescent obesity: part of a bigger picture. Lancet. (2015) 385:2510-20. doi: 10.1016/S0140-6736(14)61746-3

17. Browne NT, Snethen JA, Greenberg CS, Frenn M, Kilanowski JF, Gance-Cleveland B, et al. When pandemics collide: the impact of COVID-19 on childhood obesity. J Pediatr Nurs. (2021) 56:90-8. doi: 10.1016/j.pedn.2020.11.004

18. Ashikkali L, Carroll W, Johnson C. The indirect impact of COVID-19 on child health. Paediatr Child Health. (2020) 30:430-7. doi: 10.1016/j.paed.2020.09.004

19. Ni X, Song W, Peng X, Shen Y, Peng Y, Li Q, et al. Pediatric reference intervals in China (PRINCE): design and rationale for a large, multicenter collaborative cross-sectional study. Sci Bull. (2018) 63:1626-34. doi: 10.1016/j.scib.2018.11.024

20. WHO Multicentre Growth Reference Study Group. WHO Child Growth Standards based on length/height, weight and age. Acta Paediatr Suppl. (2006) 450:76-85. doi: 10.1111/j.1651-2227.2006.tb02378.x

21. de Onis M, Onyango AW, Borghi E, Siyam A, Nishida C, Siekmann J. Development of a WHO growth reference for school-aged children and adolescents. Bull World Health Organ. (2007) 85:660-7. doi: 10.2471/BLT.07.043497

22. Editorial Board of Chinese Journal of Pediatrics; Subspecialty Group of Child Health Care, The Society of Pediatrics, Chinese Medical Association; Subspecialty Group of Cardiovascular Disease, The Society of; Pediatrics, Chinese Medical Association; Subspecialty Group of Atherosclerosis, The Society of Cardiovascular; Disease, Chinese Medical Association. Experts consensus for prevention and treatment of dyslipidemia in children and adolescents. Zhonghua Er Ke Za Zhi. (2009) 47:426-8. (Chinese). doi: 10.3760/cma.j.issn.0578-1310.2009.06.007

23. Expert Panel on Integrated Guidelines for Cardiovascular Health and Risk Reduction in Children and Adolescents; National Heart, Lung, and Blood Institute. Expert panel on integrated guidelines for cardiovascular health and risk reduction in children and adolescents: summary report. Pediatrics. (2011) 128(Suppl 5):S213-56. doi: 10.1542/peds. 2009-2107C

24. National Kidney Foundation. K/DOQI clinical practice guidelines for bone metabolism and disease in chronic kidney disease. Am J Kidney Dis. (2003) 42(4 Suppl 3):S1-201. doi: 10.1016/S0272-6386(03)00905-3

25. Schwartz GJ, Gauthier B. A simple estimate of glomerular filtration rate in adolescent boys. J Pediatr. (1985) 106:522-6. doi: 10.1016/S0022-3476(85)80697-1

26. Schwartz GJ, Haycock GB, Edelmann CM Jr, Spitzer A. A simple estimate of glomerular filtration rate in children derived from body length and plasma creatinine. Pediatrics. (1976) 58:259-63.
27. Chou CW, Fang WH, Chen YY, Wang CC, Kao TW, Wu CJ, et al. Association between serum calcium and risk of cardiometabolic disease among community-dwelling adults in taiwan. Sci Rep. (2020) 10:3192. doi: 10.1038/s41598-020-60209-w

28. Rohrmann S, Garmo H, Malmström H, Hammar N, Jungner I, Walldius G, et al. Association between serum calcium concentration and risk of incident and fatal cardiovascular disease in the prospective AMORIS study. Atherosclerosis. (2016) 251:85-93. doi: 10.1016/j.atherosclerosis.2016.06.004

29. Saltevo J, Niskanen L, Kautiainen H, Teittinen J, Oksa H, Korpi-Hyövälti E, et al. Serum calcium level is associated with metabolic syndrome in the general population: FIN-D2D study. Eur J Endocrinol. (2011) 165:429-34. doi: 10.1530/EJE-11-0066

30. Li S, Na L, Li Y, Gong L, Yuan F, Niu Y, et al. Long-term calcium supplementation may have adverse effects on serum cholesterol and carotid intima-media thickness in postmenopausal women: a double-blind, randomized, placebo-controlled trial. Am J Clin Nutr. (2013) 98:1353-9. doi: $10.3945 /$ ajcn.113.062844

31. Vo TM, Disthabanchong S. Are there ways to attenuate arterial calcification and improve cardiovascular outcomes in chronic kidney disease? World $J$ Cardiol. (2014) 6:216-26. doi: 10.4330/wjc.v6.i5.216

32. Kim KN, Oh SY, Hong YC. Associations of serum calcium levels and dietary calcium intake with incident type 2 diabetes over 10 years: the Korean Genome and Epidemiology Study (KoGES). Diabetol Metab Syndr. (2018) 10:50. doi: 10.1186/s13098-018-0349-y

33. Rooney MR, Pankow JS, Sibley SD, Selvin E, Reis JP, Michos ED, et al. Serum calcium and incident type 2 diabetes: the Atherosclerosis Risk in Communities (ARIC) study. Am J Clin Nutr. (2016) 104:1023-9. doi: 10.3945/ajen.115.130021

34. Gallo L, Faniello MC, Canino G, Tripolino C, Gnasso A, Cuda G, et al. Serum calcium increase correlates with worsening of lipid profile: an observational study on a large cohort from south Italy. Medicine. (2016) 95:e2774. doi: 10.1097/MD.0000000000002774

35. Chen M, Xiao J, Du Y, Wang M, Ruan J, Tian Y. Elevated non-highdensity lipoprotein cholesterol corresponds to a high risk of nephrolithiasis in children. BMC Urol. (2020) 20:120. doi: 10.1186/s12894-020-00691-6

36. Päth G, Mehana AE, Pilz IH, Alt M, Baumann J, Sommerer I, et al. NUPR1 preserves insulin secretion of pancreatic $\beta$-cells during inflammatory stress by multiple low-dose streptozotocin and high-fat diet. Am J Physiol Endocrinol Metab. (2020) 319:E338-E344. doi: 10.1152/ajpendo.00088.2020

37. Xie T, So WY, Li XY, Leung PS. Fibroblast growth factor 21 protects against lipotoxicity-induced pancreatic beta-cell dysfunction via regulation of AMPK signaling and lipid metabolism. Clin Sci. (2019) 133:2029-44. doi: 10.1042/CS20190093

38. Li S, Li Y, Ning H, Na L, Niu Y, Wang M, et al. Calcium supplementation increases circulating cholesterol by reducing its catabolism via GPER and TRPC1-dependent pathway in estrogen deficient women. Int J Cardiol. (2013) 168:2548-60. doi: 10.1016/j.ijcard.2013.03.057

39. Revankar CM, Cimino DF, Sklar LA, Arterburn JB, Prossnitz ER. A transmembrane intracellular estrogen receptor mediates rapid cell signaling. Science. (2005) 307:1625-30. doi: 10.1126/science.1106943

Conflict of Interest: The authors declare that the research was conducted in the absence of any commercial or financial relationships that could be construed as a potential conflict of interest.

Publisher's Note: All claims expressed in this article are solely those of the authors and do not necessarily represent those of their affiliated organizations, or those of the publisher, the editors and the reviewers. Any product that may be evaluated in this article, or claim that may be made by its manufacturer, is not guaranteed or endorsed by the publisher.

Copyright $\odot 2021$ Peng, Hu, Nie, Cai, Yan, Liu, Cai, Song and Peng. This is an open-access article distributed under the terms of the Creative Commons Attribution License (CC BY). The use, distribution or reproduction in other forums is permitted, provided the original author(s) and the copyright owner(s) are credited and that the original publication in this journal is cited, in accordance with accepted academic practice. No use, distribution or reproduction is permitted which does not comply with these terms. 